receiving piperacillin-tazobactam (12.3\%) versus meropenem (3.7\%). ${ }^{5}$ Such data should have been discussed. Likewise, no information is provided on treatment duration or dose, which may have varied between the 2 groups in the present study. ${ }^{1}$ Both factors play a role in the outcome of treatment, especially when used against multidrug-resistant organisms. ${ }^{6}$

Interestingly, we previously showed that BSI severity or mortality among spinal cord injury patients over 15 years was not related to the multidrug-resistant characteristics of the microorganism. ${ }^{7}$ Although our sample size was small $(\mathrm{n}<30)$, a closer look at the outcome between ESBL-EC $(n=26)$ and ESBL-KP $(\mathrm{n}=13)$ did not reveal any statistical difference in terms of mortality rate $(7.7 \%$ in each arm). Moreover, the mortality rates were similar for other ESBL microorganisms (Enterobacter spp, Morganella spp, and Proteus spp $(\mathrm{n}=21)), \sim 9.5 \%(P=.99$, data not shown).

In fact, we believe that the findings of Scheuerman et al, which showed no impact of CTX-M isolates in comparison to other ESBL genotypes, might support the idea that the type of germ does not play a major role. Indeed, mortality seems more related to patient comorbidities and severity of infection, as shown in Table 2 of the article, ${ }^{1}$ with significant discrepancies between the 2 groups in terms of length of stay to bacteremia $(P=.017)$, source of infection $(P=.005)$, ICU ward admission $(P<.001)$ and underlying cardiovascular disease $(P<.001)$. Moreover, in a rabbit model of sepsis induced by a multidrug-resistant Klebsiella pneumoniae, Zhou et $\mathrm{al}^{8}$ showed that mortality was higher for the rabbits infected by susceptible than those infected with multidrug-resistant strains.

Overall, the impact of ESBL-KP isolates on mortality rate might have been overestimated, in the light of the severity of the patient condition.
Financial support. No financial support was provided relevant to this article.

Potential conflicts of interest. All authors report no conflicts of interest relevant to this article.

\section{References}

1. Scheuerman O, Schechner V, Carmeli Y, et al. Comparison of predictors and mortality between bloodstream infections caused by ESBL-producing Escherichia coli and ESBL-producing Klebsiella pneumoniae. Infect Control Hosp Epidemiol 2018;39:660-667.

2. Tabah A, Koulenti D, Laupland $\mathrm{K}$, et al. Characteristics and determinants of outcome of hospital-acquired bloodstream infections in intensive care units: the EUROBACT International Cohort Study. Intensive Care Med 2012;38:1930-1945.

3. Adrie C, Garrouste-Orgeas M, Ibn Essaied W, et al. Attributable mortality of ICU-acquired bloodstream infections: impact of the source, causative micro-organism, resistance profile and antimicrobial therapy. I Infect 2017;74:131-141.

4. Artero A, Esparcia A, Eiros JM, Madrazo M, Alberola J, Nogueira JM. Effect of bacteremia in elderly patients with urinary tract infection. Am J Med Sci 2016;352:267-271.

5. Harris PN, Peleg AY, Iredell J, et al. Meropenem versus piperacillintazobactam for definitive treatment of bloodstream infections due to ceftriaxone non-susceptible Escherichia coli and Klebsiella spp (the MERINO trial): study protocol for a randomised controlled trial. Trials 2015;16:24.

6. Davido B, Bouchand F, Dinh A, et al. Reinforcement of an antimicrobial stewardship task force aims at a better use of antibiotics of last resort: the COLITIFOS study. Int J Antimicrob Agents 2017;50:142-147.

7. Saliba M, Saadeh D, Bouchand F, et al. Outcome of bloodstream infections among spinal cord injury patients and impact of multidrug-resistant organisms. Spinal Cord 2017;55(2). doi: 10.1038/sc.2016.176.

8. Zhou Z, Ren J, Liu H, Gu G, Li J. Pandrug-resistant isolate of Klebsiella pneumoniae causes less damage than drug-susceptible isolates in a rabbit model. Clin Invest Med 2011;34:E38-E44.

\title{
Differences in mortality between infections due to extended- spectrum-beta-lactamase-producing Klebsiella pneumoniae and Escherichia coli
}

\author{
Jason P. Burnham $\mathrm{MD}^{1}$, Jennie H. Kwon DO, $\mathrm{MSCl}^{1}$, Margaret A. Olsen PhD, $\mathrm{MPH}^{1}$, Hilary M. Babcock MD, $\mathrm{MPH}^{1}$ \\ and Marin H. Kollef MD² \\ ${ }^{1}$ Division of Infectious Diseases Washington University School of Medicine, St Louis, Missouri; and ${ }^{2}$ Division of Pulmonary and Critical Care Medicine, \\ Washington University School of Medicine, St Louis, Missouri.
}

To the Editor-We read with interest the recent article by Scheuerman et al, ${ }^{1}$ in which they found that patients with

Author for correspondence: Jason P. Burnham, MD, Division of Infectious Diseases Medicine, Washington University School of Medicine, 4523 Clayton Avenue, Campus Box 8051, St Louis, MO 63110. E-mail: burnham@wustl.edu or Marin H. Kollef, MD, Division of Pulmonary and Critical Care, Washington University School of Medicine, 4523 Clayton Avenue, Campus Box 8052, St Louis, MO 63110. E-mail: kollefm@wustl. edu

Cite this article: Burnham JP, et al. (2018). Differences in mortality between infections due to extended-spectrum-beta-lactamase-producing Klebsiella pneumoniae and Escherichia coli. Infection Control \& Hospital Epidemiology 2018, 39, 1138-1139. doi: 10.1017/ice.2018.142 extended-spectrum- $\beta$-lactamase (ESBL) producing Klebsiella pneumoniae infections had higher 30-day mortality than patients with ESBL producing Escherichia coli infections. We have recently published on mortality, readmissions, recurrences, and the benefit of infectious diseases consultation for patients with various multidrug resistant organism infections. ${ }^{2,3}$ We included in our study patients with various ESBL producing Enterobacteriaceae infections, among them K. pneumoniae and E. coli. Given the recent findings of Scheuerman et al, we conducted a retrospective evaluation to determine the association between ESBL producing organism (K. pneumoniae or E. coli) and 30-day all-cause mortality at our institution. 
Table 1. Factors Associated with 30-Day All-Cause Mortality in a Cox Proportional Hazards Model ${ }^{\mathrm{a}}$

\begin{tabular}{lcc}
\hline Risk Factor & $\begin{array}{c}\text { Hazard Ratio } \\
(95 \% \mathrm{CI})\end{array}$ & $\begin{array}{c}P \\
\text { Value }\end{array}$ \\
\hline $\begin{array}{l}\text { Infection with ESBL producing K. pneumoniae } \\
\text { (reference group E. coli) }\end{array}$ & $1.69(0.97-2.92)$ & .062 \\
\hline Comorbidity & $3.20(1.75-5.82)$ & $<.001$ \\
\hline Metastatic cancer & $6.19(2.38-15.1)$ & $<.001$ \\
\hline HIV/AIDS & $2.11(1.38-3.46)$ & .001 \\
\hline Cardiovascular disease & $0.68(0.42-1.08)$ & .100 \\
\hline Chronic respiratory failure & $4.95(3.17-7.75)$ & $<.001$ \\
\hline Cirrhosis & $2.42(1.34-4.38)$ & .003 \\
\hline Leukemia & $2.00(1.23-3.26)$ & .005 \\
\hline Solid organ cancer & & \\
\hline Index hospitalization characteristics & $0.37(0.22-0.60)$ & $<.001$ \\
\hline ID consultation & $2.87(1.77-4.64)$ & $<.001$ \\
\hline Required ICU care during hospitalization & $2.29(1.31-3.99)$ & .003 \\
\hline APACHE II score $>21$ & & \\
\hline
\end{tabular}

NOTE. HIV/AIDS, human immunodeficiency virus/acquired immunodeficiency syndrome; APACHE, Acute Physiology and Chronic Health Evaluation; $\mathrm{Cl}$, confidence interval; HR, hazard ratio; ICU, intensive care unit; ID, infectious diseases.

${ }^{a}$ Variables not retained in final model included admitting service, sex, lymphoma, congestive heart failure, diabetes, solid organ transplant, bone marrow transplant, chronic kidney disease, and end-stage renal disease.

This study was conducted at Barnes-Jewish Hospital, a 1,250bed academic medical center in St Louis, Missouri. The study period was January 1, 2006, to October 1, 2015. We included patients with positive sterile site and bronchial wash/bronchoalveolar lavage cultures for ESBL-producing E. coli and K. pneumoniae. Sterile sites were defined as bloodstream; pleural, intra-abdominal, pericardial, cerebrospinal, and synovial fluids; bone marrow; and surgical specimens collected from lymph nodes, central nervous system, liver, spleen, kidney, pancreas, ovary, or vascular tissue. The presence of ESBL production was assumed based on ceftriaxone, aztreonam, cefotaxime, or ceftazidime nonsusceptibility. ${ }^{4}$ The Washington University School of Medicine Institutional Review Board approved this study with a waiver of informed consent.

The primary endpoint was 30-day all-cause mortality. KaplanMeier curves for 30-day all-cause mortality were generated to compare organism type and significance determined using the log-rank test. Escherichia coli was used as the reference group for determining the association between organism and mortality. Log-log survival plots were used to graphically test the proportional hazards assumption. Factors associated with mortality in bivariate analysis $(P<.20)$ were entered into a multivariate Cox proportional hazards model to determine hazard ratios (HR) for 30-day all-cause mortality. All analyses were conducted with SPSS version 25 software (IBM, Armonk, NY).

In total, 605 patients met the eligibility criteria: 543 with ESBL E. coli and 62 with ESBL $K$. pneumoniae. Among patients with ESBL E. coli, 96 (17.7\%) died within 30 days compared to 16 (25.8\%) with ESBL K. pneumoniae. In Kaplan-Meier analysis, mortality was not significantly different between patients infected with $K$. pneumoniae and E. coli ESBL organisms $(P=0.12)$. Variables retained in the final Cox proportional hazards model are shown in Table 1. Infection with ESBL-producing $K$. pneumoniae was marginally associated with mortality in the final Cox proportional hazards model (hazard ratio [HR], 1.69; 95\% confidence interval [CI], 0.97-2.92; $P=.062$ ). Because the number of patients in the ESBL $K$. pneumoniae group was small, we performed a post-hoc power analysis and found that we had only $35.8 \%$ power to detect a significant difference in mortality.

Our results add to the body of literature on differences in mortality between ESBL-producing K. pneumoniae and E. coli. Despite the low power of our study, ESBL-producing $K$. pneumoniae was associated with marginally increased risk of 30-day mortality. Our data are important to add to the literature on this subject and could be combined with the data of Scheuerman et al and future studies in a meta-analysis to more definitively determine the association between ESBL-producing organism type and mortality. In addition, as demonstrated in our previous study, infectious disease consultation was associated with reduced mortality for drug-resistant Enterobacteriaceae infections.

Acknowledgments. The content is solely the responsibility of the authors and does not necessarily represent the official view of the National Institutes of Health (NIH).

Financial support. This work was supported by a grant from the Washington University Institute of Clinical and Translational Sciences (grant no. UL1TR000448) and by a subaward (no. KL2TR000450) from the National Center for Advancing Translational Sciences (NCATS) of the NIH.

Potential conflicts of interest. Dr Kollef was supported by the BarnesJewish Hospital Foundation. All other authors report no conflicts of interest relevant to this article.

\section{References}

1. Scheuerman O, Schechner V, Carmeli Y, et al. Comparison of predictors and mortality between bloodstream infections caused by ESBL-producing Escherichia coli and ESBL-producing Klebsiella pneumoniae. Infect Control Hosp Epidemiol 2018;39:660-667.

2. Burnham JP, Kwon JH, Olsen MA, Babcock HM, Kollef MH. Readmissions with multidrug-resistant infection in patients with prior multidrugresistant infection. Infect Control Hosp Epidemiol 2018;39:12-19.

3. Burnham JP, Olsen MA, Stwalley D, Kwon JH, Babcock HM, Kollef MH. Infectious diseases consultation reduces 30-day and 1-year all-cause mortality for multidrug-resistant organism infections. Open Forum Infect Dis 2018;5(3):ofy026.

4. Laboratory detection of extended-spectrum $\beta$-lactamases (ESBLs). Centers for Disease Control and Prevention website. https://www.cdc.gov/hai/ settings/lab/lab_esbl.html. Published 2010. Accessed May 30, 2018. 